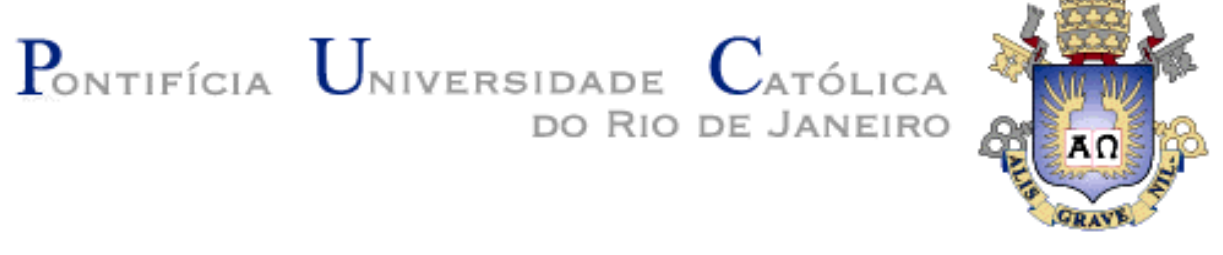

Ronald Rojas Hacha

\title{
Caracterização Mineralógica do Minério de Ouro da Rio Paracatu Mineração (RPM), visando a determinação de minerais portadores de titânio.
}

\section{Dissertação de Mestrado}

Dissertação apresentada como requisito parcial para obtenção do título de Mestre pelo Programa de Pós-Graduação em Engenharia de Materiais e de Processos Químicos e Metalúrgicos do Departamento de Engenharia de Materiais da PUC - Rio.

Orientadores: Prof. Maurício Leonardo Torem Co-orientador: Prof. Luiz Carlos Bertolino

Rio de Janeiro

Agosto de 2010 


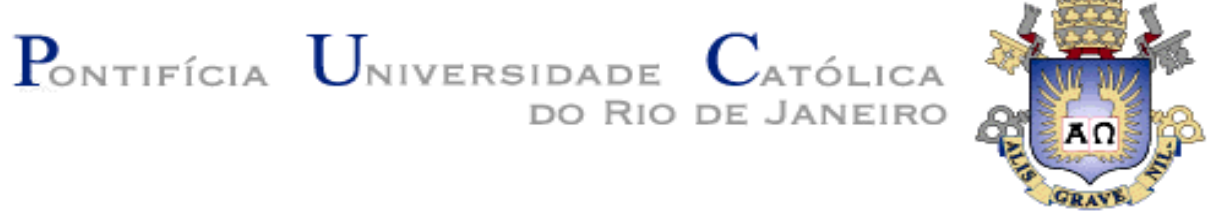

Ronald Rojas Hacha

\section{Caracterização Mineralógica do Minério de Ouro da Rio Paracatu Mineração (RPM), visando a determinação de minerais portadores de titânio.}

Dissertação apresentada como requisito parcial para obtenção do título de Mestre pelo Programa de Pós-Graduação em Engenharia de Materiais e de Processos Químicos e Metalúrgicos do Departamento de Engenharia de da PUC Rio. Aprovada pela Comissão Examinadora abaixo assinada.

Prof. Maurício Leonardo Torem

Orientador

Departamento de Engenharia de Materiais - PUC - Rio

Prof. Luiz Carlos Bertolino

Co-orientador

Centro de Tecnologia Mineral - CETEM / MCT

Prof. Marisa Bezerra de Mello Monte Centro de Tecnologia Mineral - CETEM / MCT

Prof. Otávio da Fonseca Martins Gomes Centro de Tecnologia Mineral - CETEM / MCT

Prof. Francisco José Moura Departamento de Engenharia de Materiais - PUC - Rio

Prof. José Eugenio Leal Coordenador (a) Setorial do Centro Técnico Científico - PUC - Rio 
Todos os direitos reservados. É proibida a reprodução total ou parcial do trabalho sem autorização da universidade, do autor e do orientador.

\section{Ronald Rojas Hacha}

Graduou-se em Engenharia Química na Universidade Nacional de San Agustín de Arequipa (Peru) em 2004. Obteve o título de Engenheiro Químico pela Universidade Nacional de San Agustín de Arequipa em 2007.

Ficha Catalográfica

Rojas, Ronald Hacha.

Caracterização mineralógica do minério de ouro da Rio Paracatu Mineração (RPM), visando a determinação de minerais portadores de titânio / Ronald Rojas Hacha; orientadores: Maurício Leonardo Torem; co-orientador: Luiz Carlos Bertolino. - 2010.

118 f. : il. (color.) ; $30 \mathrm{~cm}$

Dissertação (Mestrado)-Pontifícia Universidade Católica do Rio de Janeiro, Departamento de Ciência dos Materiais e Metalurgia, 2010.

Incluí bibliografia.

1. Ciência dos Materiais e Metalurgia - Teses. 2. Caracterização mineral. 3. Titânio. 4. Liberação. I. Torem, Maurício Leonardo. II. Bertolino, Luiz Carlos. III. Pontifícia Universidade Católica do Rio de Janeiro. Departamento de Ciência dos Materiais e Metalurgia. III. Título. 
Para meus pais Agustino e Alejandrina, pela vida, amor e educação, para meus irmãos pelo amor e amizade. 


\section{Agradecimentos}

A Deus por me amparar nos momentos difíceis, me dar força interior para superar as dificuldades e mostrar os caminho nas horas incertas.

Ao Professor Doutor Maurício Leonardo Torem, meu orientador, pela confiança, paciência e compreensão oferecidos durante a orientação.

Ao Professor Doutor Luiz Carlos Bertolino, meu co-orientador, pela competência científica e acompanhamento do trabalho, pela disponibilidade e generosidade reveladas ao longo do trabalho, assim como pelas críticas, correções, sugestões e amizade então demonstradas.

À Doutora Marisa Bezerra de Mello Monte, pela permanente disponibilidade, me permitindo encontrar informações e soluções que em muito contribuíram para a execução desta dissertação.

Aos Doutores Otávio da Fonseca Martins Gomes e Reiner Neumann, pelo apoio prestado na realização do estudo de caracterização com o MLA.

À CAPES pela bolsa concedida durante a realização deste mestrado, o que contribuiu para a concretização desta dissertação.

Ao CETEM/MCT pela oportunidade e apoio para a realização deste trabalho. 
Ao Departamento de Engenharia de Materiais (DEMa) da PUC - Rio, seus professores, pesquisadores e funcionários.

Aos meus colegas do Mestrado, pela excelente relação pessoal que criamos e que espero que não se perca e pela ajuda e intercâmbio de idéias e informação para a elaboração deste trabalho, pelas informações prestadas por toda a colaboração, apoio e amizade.

Aos meus amigos, Fernanda, Carla, Adriana, Mirella, Diana, Antonio, Julio e Diego, pela disponibilidade, colaboração, e amizade demonstradas na culminação desta dissertação.

A Adauto, Edivaldo, Vagner, Felipe, Edinaldo, Patrik, Antonieta e Jackson, técnicos do CETEM/MCT, pela ajuda prestada na preparação e análises das amostras.

Agradeço ao Grupo Kinross e a Rio Paracatu Mineração pelo apoio financeiro oferecido para o desenvolvimento deste trabalho na pessoa do diretor de exploração da RPM Brian Tomson.

A minha família adotiva no Brasil Abbud, Alex, Jane, Jean, Raquel, Jennifer e Jonathan. 


\section{Resumo}

Rojas, Ronald Hacha; Torem, Mauricio Leonardo. Caracterização Mineralógica do minério de Ouro da Rio Paracatu Mineração (RPM), visando a determinação de minerais de titânio. Rio de Janeiro, 2010, 118p. Dissertação de Mestrado - Departamento de Ciência dos Materiais e Metalurgia, Pontifícia Universidade Católica do Rio de Janeiro.

Neste trabalho são apresentados os resultados da caracterização mineralógica de uma amostra de minério de ouro da RPM - Kinross, que teve como objetivo principal, identificar os minerais portadores de titânio e avaliar o espectro de liberação dos minerais de interesse. A metodologia empregada compreendeu a realização de análises granulométricas, separações em meio denso e magnética. Os estudos mineralógicos foram realizados por meio da difratometria de raios $\mathrm{X}(\mathrm{DRX})$, microscopia ótica e microscopia eletrônica de varredura (MEV) por meio do sistema "Mineral Liberation Analyzer" - MLA. Os produtos obtidos foram submetidos à análise química por espectrometria de fluorescência de raios $\mathrm{X}(\mathrm{FRX})$. As análises químicas indicaram que a amostra estava constituída essencialmente por $\mathrm{SiO}_{2}(66,4 \%), \mathrm{Al}_{2} \mathrm{O}_{3}(14,2 \%), \mathrm{Fe}_{2} \mathrm{O}_{3}(7,22 \%)$ e $\mathrm{TiO}_{2}(1 \%)$. Visando avaliar o espectro de liberação dos minerais portadores de titânio, o estudo foi focado em seis faixas granulométricas $(-300+212 ;-212+150$; $150+104 ;-104+74 ;-74+53$; e $-53+37 \mu \mathrm{m})$. Cerca de $20 \%$ do material de todas as frações foi constituída por material afundado (meio denso), sendo composto principalmente de $\mathrm{SiO}_{2}$ (35\%), $\mathrm{Fe}_{2} \mathrm{O}_{3}(30 \%), \mathrm{Al}_{2} \mathrm{O}_{3}(>7 \%)$ e $\mathrm{TiO}_{2}(<5 \%)$. A fração flutuada é composta em sua maioria de $\mathrm{SiO}_{2}$ e $\mathrm{Al}_{2} \mathrm{O}_{3}$. As análises de DRX da fração afundada indicaram a presença de ilmenita, anatásio e rutilo. As frações afundadas foram submetidas à separação magnética no separador Frantz em diferentes intensidades de corrente ( 0,3 até $1,75 \mathrm{~A})$, através desta operação se concentrou até $8 \%$ em massa de $\mathrm{TiO}_{2}$ na fração $-104+74 \mu$ m e a $0,5 \mathrm{~A}$. As frações afundadas foram submetidas a estudos sistemáticos no MEV com o sistema MLA, confirmando a presença de ilmenita, anatásio e rutilo como os minerais portadores de titânio. A liberação completa dos minerais carreadores de titânio foi 
aproximadamente de $1 \%$ em massa, já a ganga liberou-se mais de $90 \%$ em massa.

A partir dos resultados obtidos se observou que é possível concentrar o $\mathrm{TiO}_{2}$ contido no minério.

\section{Palavras chave}

Caracterização mineralógica; liberação; titânio. 


\section{Abstract}

Rojas, Ronald Hacha; Torem, Mauricio Leonardo (Advisor). Mineralogical Characterization of Gold Ore the Rio Paracatu Mineração (RPM), aiming at the determination of Titanium-Bearing Minerals. Rio de Janeiro, 2010, 118p. MSc. Dissertation - Departamento de Ciência dos Materiais e Metalurgia, Pontifícia Universidade Católica do Rio de Janeiro.

This work presents studies in Minerals Characterization of gold ore sample from RPM-Kinross with objective to identify their titanium-bearing minerals and to assess its behavior in different size fraction (spectrum release). The methodology involved particle size analysis and minerals separation (separation in dense medium and magnetic separation), followed of mineralogical studies by $\mathrm{X}$ Ray Diffraction, Optical Microscopy and Scanning Electron Microscopy (SEM) by using the Mineral Liberation Analyzer-MLA. The products obtained were submitted to Chemical Analysis of X-Ray Fluorescence. The analysis of X-Ray Fluorescence revealed that the sample studied was formed essentially by $\mathrm{SiO}_{2}$ (66,4\%), $\mathrm{Al}_{2} \mathrm{O}_{3}(14,2 \%), \mathrm{Fe}_{2} \mathrm{O}_{3}(7,22 \%)$ and $\mathrm{TiO}_{2}(1 \%)$. The studies were focused in six different sizes $(-300+212 ;-212+150 ;-150+104 ;-104+74 ;-74+53$ and $53+37 \mu \mathrm{m})$. ). About $20 \%$ of the material from all fractions material is sunk (dense medium), composed mainly of $\mathrm{SiO}_{2}(35 \%), \mathrm{Fe}_{2} \mathrm{O}_{3}(30 \%), \mathrm{Al}_{2} \mathrm{O}_{3}(>7 \%)$ and $\mathrm{TiO}_{2}$ $(<5 \%)$. The floated fraction was composed mainly of $\mathrm{SiO} 2$ and $\mathrm{A} 12 \mathrm{O} 3$. The XRD of the sunken fraction indicated the presence of ilmenite, rutile and anatase. The sunken fractions were subjected to magnetic separation in the Frantz separator at different current intensities ( 0.3 to $1.75 \mathrm{~A}$ ), this operation was concentrated up to 8 wt $\% \mathrm{TiO}_{2}$ in the fraction $-104+74 \mu \mathrm{m}$ and $0.5 \mathrm{~A}$. The sunken fractions were subjected to systematic studies in the SEM system with MLA, confirming the presence of ilmenite, rutile and anatase as the titanium-bearing minerals. The gangue has been release of the mineral carrier of titanium was approximately $1 \%$ wt $\%$, the denim has released more than $90 \%$ wt $\%$. From the results it was observed that it is possible to concentrate the $\mathrm{TiO}_{2}$ contained in the ore.

\section{Keywords}

Mineralogical characterization; liberation; titanium. 


\section{Sumário}

1 Introdução

2 Objetivos e Relevância do Trabalho 21

2.1. Objetivo Geral 22

2.2. Objetivos Específicos 22

3 Revisão Bibliográfica $\quad 23$

3.1. Titânio 23

3.1.1. Geologia dos Depósitos de Titânio 23

3.1.2. Reservas e Concentrados de Titânio 27

3.1.3. Dióxido de Titânio $\left(\mathrm{TiO}_{2}\right)$

3.2. Rio Paracatu Mineração (RPM) 32

3.2.1. Descrição geral das instalações de processo da RPM 33

3.2.2. Geologia do Depósito de Ouro da RPM 36

3.3. Caracterização mineralógica 40

3.3.1. Separação em meio denso 40

3.3.2. Separação magnética 41

3.3.3. Difratometria de raios X (DRX) 41

3.3.4. Microscopia Ótica (MO) 43

3.3.5. Espectrometria de Fluorescência de Raios X (FRX) 43

3.3.6. Microscopia Co-localizada 44

3.3.7. Automatização da Microscopia Eletrônica de Varredura (MEV) baseada no Sistema de Liberação Mineral 44

3.3.8. Mineral Liberation Analizer - MLA 48

3.3.9. Análises de imagens digitais 53

3.3.10. Liberação mineral 54

4 Materiais e Métodos $\quad 57$

4.1. Amostragem e Preparação 58

4.2. Análise granulométrica 59 
4.3. Separação em meio denso $\quad 59$

4.4. Separação magnética - Separador Frantz 60

4.5. Análise por Difratometria de Raios X (DRX) 62

4.6. Análise por Espectrometria de Fluorescência de Raios $X$ (FRX) 62

4.7. Preparação de amostras para Microscopia (MO e MEV) 62

4.8. Microscopia ótica aquisição de imagens 63

4.9. Aquisição e Processamento de dados de MEV - MLA 64

5 Resultados e Discussões 66

5.1. Distribuição granulométrica a úmido 66

5.2. Ensaios de separação em meio denso 69

5.3. Ensaios de separação magnética 70

5.4. Técnicas de caracterização 71

5.4.1. Lupa Binocular 71

5.4.2. Análise por Difração de Raios $X$

5.4.3. Análise química por Espectrometria de Fluorescência de Raios X $\quad 80$

5.4.4. Microscopia Co-localizada MO-MEV 87

5.4.5. Mineral Liberation Analyzer (MLA) 90

$\begin{array}{ll}6 \text { Conclusões } & 107\end{array}$

7 Referencias Bibliográficas $\quad 108$

8 Anexos 115 


\section{Lista de figuras}

Figura 1 - Mapa de operações da Rio Paracatu Mineração. Fonte: $<$ http://www.rioparacatumineracao.com.br> acesso em 01/10/09.

Figura 2 - Fluxograma de Processo - Moagem. Fonte: RPM. 34

Figura 3 - Fluxograma de Processo - Flotação. Fonte: RPM. 35

Figura 4 - Fluxograma simplificado - Remoagem e Espessamento. Fonte: RPM

Figura 5 - Mapa geológico e posicionamento estratigráfico das unidades Proterozóicas da Faixa de Dobramentos Brasília. (modificado de FUCK, et al. 1994).

Figura 6 - Mapa geológico da Rio Paracatu Mineração (modificado do Serviço Geográfico do Ministério do Exército/1972).

Figura 7 - Diagrama de aquisição de imagens usada pelo sistema MLA. \&: Papel fundamental do analisador na toma de decisões.

Figura 8 - Identificação de minerais: MLA ParticleX.

Figura 9 - Processamento de imagens: MLA Image Processing Tool.

Figura 10 - Base de dados: MLA Mineral Database Maker 2008.

Figura 11 - Ilustração geral da variação do teor limite liberação/curva de recuperação. Adaptação de MILLER, et al., 2009.

Figura 12 - Fluxograma utilizado na caracterização mineralógica do minério da RPM.

Figura 13 - Fluxograma detalhado utilizado na preparação da amostra.

Figura 14 - Peneiramento a úmido. (A) Peneiramento de finos, (B) Série de peneira Tyler.

Figura 15 - Ensaio de separação em meio denso. (A) Funil de separação, (B) Descarga do material. 
Figura 16 - Separador magnético Frantz. (A) Separação magnética, $(B)$ descarga do material magnético e não magnético.

Figura 17 - Em (A), amostras embutidas, em (B), amostras polidas recobertas com carbono, em (C), banho ultrassônico câmera de vácuo e câmera de pressão, em (D), politriz automático Struers Tegra.

Figura 18 - Microscópio Eletrônico de Varredura FEI Quanta 400.

Figura 19 - Fluxograma de processamento de dados Sistema MLA.

Figura 20 - Representação gráfica dos resultados da distribuição granulométrica.

Figura 21 - Representação gráfica dos resultados da distribuição granulométrica apos a britagem no britador de mandíbulas.

Figura 22 - Representação gráfica dos resultados obtidos na separação em meio denso.

Figura 23 - Representação gráfica dos resultados obtidos na separação magnética.

Figura 24 - Fotografias do minério, em (A), fração $-600+425 \mu \mathrm{m}$, em (B), fração $-3400+2400 \mu \mathrm{m}$, em (C) e (D), fração $1700+1200 \mu \mathrm{m}$. Notação utilizada: Qz - quartzo, Msc muscovita, Cal - calcopirita, Sd - siderita, III - illita, Fdp Feldspato.

Figura 25 - Fotografia do produto da separação em meio denso, em (A) material flutuado, em (B), material afundado, as duas figuras são frações de $-300+212 \mu \mathrm{m}$. Notação utilizada; Qz: Quartzo, Sd: Siderita, Fdp: feldspato.

Figura 26 - Fotografia da separação magnética. Em (A), material de fração $-300+212 \mu \mathrm{m}$ susceptível a 0,3 A., em (B) material de fração de -104+74 $\mu$ m susceptível a 0,30 A. Notação utilizada; Qz: Quartzo, Sd: Siderita.

Figura 27 - Fotografia da separação magnética. Em (A) e (B) 
material susceptível a 0,5A de uma fração de $-104+74$

$\mu \mathrm{m}$. Notação utilizada; Qz: Quartzo, Sd: Siderita, Msc:

Muscovita, IIm: IImenita.

Figura 28 - Difratogramas de raios $X$ da amostra do minério da RPM. Radiação Co Ka (40 kV/40 mA).

Figura 29 - Difratogramas de raios $X$ de frações grossa e fina do minério da RPM. Radiação Co Ka (40 kV/40 mA).

Figura 30 - Difratogramas de raios $X$ das amostras obtidas na separação em meio denso. Radiação Co Ka (40 kV/40 $m A)$.

Figura 31 - Difratogramas de raios $X$ da amostra do minério de ouro da RPM, fração $-37 \mu \mathrm{m}$. Radiação Co Ka (40 $\mathrm{kV} / 40 \mathrm{~mA}$ ).

Figura 32 - Difratogramas de raios $X$ de uma fração de $212+150 \mu \mathrm{m}$, a diferentes intensidades de corrente. Radiação Co Ka (40 kV/40 mA).

Figura 33 - Difratogramas de raios $X$ de uma fração de $150+104 \mu \mathrm{m}$ a diferentes intensidades de corrente. Radiação Co Ka (40 kV/40 mA).

Figura 34 - Difratogramas de raios $X$ de uma fração de $104+74 \mu \mathrm{m}$ a diferentes intensidades de corrente. Radiação Co Ka $(40$ kV/40 mA).

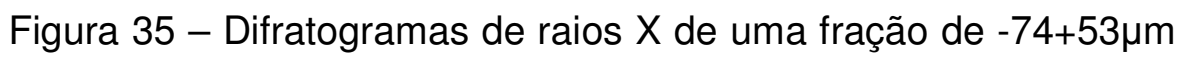
a diferentes intensidades de corrente. Radiação Co Ka (40 kV/40 mA).

Figura 36 - Composição química do material afundado.

Figura 37 - Distribuição do $\mathrm{TiO}_{2}$ obtidas na separação magnética.

Figura 38 - Gráfico comparativo da distribuição do $\mathrm{TiO}_{2}$ na separação magnética em diferentes granulométricas e em intensidades de corrente.

Figura 39 - Porcentagem de $\mathrm{TiO}_{2}$ presente no material magnético para uma intensidade de corrente 0,3A das diferentes frações separadas. 
Figura 40 - Imagem obtida no microscópio óptico.

Figura 41 - Comparação de fases: (A) tabela de cores do MLA; (B) imagem de MEV (BSE); (C) imagem de MLA; (D) imagem de MO.

Figura 42 - Apresentação de alguns minerais que foram segmentados (fração $-150+104 \mu \mathrm{m}$ ): MLA Image Processing Tool.

Figura 43 - Microfotografia da ilmenita obtida no MEV.

Figura 44 - Microfotografia da ilmenita após a segmentação no MLA.

Figura 45 - Microfotografia do rutilo obtida no MEV.

Figura 46 - Microfotografia do rutilo após a segmentação no MLA.

Figura 47 - Composição mineralógica do material afundado na separação no meio denso.

Figura 48 - Comportamento da ilmenita e o quartzo em diferentes frações

Figura 49 - Associação mineralógica da ilmenita na separação em meio denso realizada no minério total (fração granulométrica: $-300+37 \mu \mathrm{m})$.

Figura 50 - Associação mineralógica da ilmenita na fração $150+104 \mu \mathrm{m}$.

Figura 51 - Associação mineralógica da ilmenita na fração -

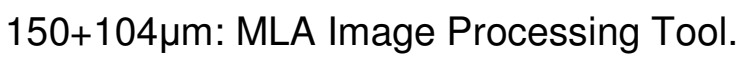

Figura 52 - Associação mineralógica do anatásio na separação em meio denso realizada para o minério total.

Figura 53 - Associação mineralógica do anatásio na fração 104+74 $\mu \mathrm{m}$ : MLA Image Processing Tool.

Figura 54 - Variação da liberação - teor limite/curva de recuperação da ilmenita para uma faixa de tamanho de $-300+37 \mu \mathrm{m}$.

Figura 55 - Variação da liberação - teor limite/curva de recuperação para a ilmenita em diferentes frações.

Figura 56 - Variação da liberação - teor limite/curva de 
recuperação para o anatásio em diferentes frações.

Figura 57 - Distribuição da liberação de partículas de ilmenita em função à composição para uma faixa de tamanho de $300+37 \mu \mathrm{m}$.

Figura 58 - Distribuição da liberação de partículas de ilmenita em função à composição em diferentes frações granulométricas.

Figura 59 - Distribuição da liberação de partículas de ilmenita em função à composição em diferentes frações com um corte no limite da escala do eixo $\mathrm{Y}$ de $10 \%$.

Figura 60 - Distribuição da liberação de partículas de anatásio em função á composição para uma faixa de tamanho de $300+37 \mu \mathrm{m}$.

Figura 61 - Distribuição da liberação de partículas de para a composição de anatásio em diferentes frações granulométricas.

Figura 62 - Distribuição da liberação de partículas de anatásio em função à composição em diferentes granulométricas com um corte no limite na escala do eixo $Y$ de $10 \%$.

Figura 63 - Tipo de liberação para a ilmenita.

Figura 64 - Tipo de liberação para o anatásio. 


\section{Lista de tabelas}

Tabela 1 - Composição química do minério Bulk da RPM. 20

Tabela 2 - Conteúdos típicos e proporções de titânio em algumas rochas comuns. 24

Tabela 3 - Composição de alguns minerais de titânio comuns. 26

Tabela 4 - Tipos de depósitos de minerais de titânio e seus minerais. 26

Tabela 5 - Reserva e produção mundial de minerais de titânio. 28

Tabela 6 - Distribuição granulométrica do minério da RPM (alimentação SAG). $\quad 67$

Tabela 7 - Distribuição granulométrica após a britagem. 68

Tabela 8 - Resultados do Afunda - Flutua com o uso de bromofórmio $\left(\mathrm{CHBr}_{3}\right)$

Tabela 9 - Resultados da separação magnética realizada no separador Frantz (\% em massa). $\quad 70$

Tabela 10 - Composição química do material afundado no meio denso (\% em massa). 80

Tabela 11 - Composição química (\% em massa) da fração $300+212 \mu \mathrm{m}$. 82

Tabela 12 - Composição química (\% em massa) da fração $212+150 \mu \mathrm{m}$.

Tabela 13 - Composição química (\% em massa) da fração $150+104 \mu \mathrm{m}$.

Tabela 14 - Composição química (\% em massa) da fração $104+74 \mu \mathrm{m}$.

Tabela 15 - Composição química (\% em massa) da fração $74+53 \mu \mathrm{m}$.

Tabela 16 - Composição química (\% em massa) da fração $53+37 \mu \mathrm{m}$.

Tabela 17 - Resultados da separação de \% em massa do $\mathrm{TiO}_{2}$ na separação magnética. 
Tabela 18 - Alguns minerais presentes no minério de ouro da RPM.

Tabela 19 - Composição mineralógica do minério da RPM em diferentes frações. 93

Tabela 20 - Composição elementar do minério da RPM 95 